\title{
Validity of the One and One-Half Layer Quasi-Geostrophic Model and Effective Topography
}

\author{
ANDREW MAJDA ${ }^{1}$ AND XIAOMING WANG ${ }^{2}$ \\ ${ }^{1}$ Courant Institute of Mathematics Sciences, New York University, \\ New York, USA \\ ${ }^{2}$ Department of Mathematics, Florida State University and \\ Iowa State University, Tallahassee, Florida, USA
}

\begin{abstract}
We rigorously justify the so-called one and one-half layer quasi-geostrophic model from the two layer model as the ratio of the depth of the bottom layer over that of the top layer approaches infinity. The effective dynamics is given by the classical barotropic quasi-geostrophic dynamics for the bottom layer without topography, and the one layer quasi-geostrophic dynamics with the stream function of the bottom layer serving as an effective (possibly time-dependent) topography for the the top layer. Such a one and one-half layer model is utilized in successful quantitative prediction of the Great Red Spot on Jupiter (see Turkington et al., 2001).
\end{abstract}

Keywords Effective topography; One and one-half layer model; Quasigeostrophic model; Two-layer model.

Mathematics Subject Classification 35Q35; 35B20; 76B99.

\section{Introduction}

One of the simplest useful models in geophysical fluid dynamics that takes into consideration rotation and stratification is the two layer model for quasi-geostrophic flows (see Gill, 1982; Pedlosky, 1987, for instance):

$$
\begin{aligned}
& \frac{\partial}{\partial t}\left(\Delta \psi_{1}-F_{1}\left(\psi_{1}-\psi_{2}\right)+\beta y\right)+J\left(\psi_{1}, \Delta \psi_{1}-F_{1}\left(\psi_{1}-\psi_{2}\right)+\beta y\right)=\mathscr{F}_{1}, \\
& \frac{\partial}{\partial t}\left(\Delta \psi_{2}-F_{2}\left(\psi_{2}-\psi_{1}\right)+\beta y\right)+J\left(\psi_{2}, \Delta \psi_{2}-F_{2}\left(\psi_{2}-\psi_{2}\right)+\beta y\right)=\mathscr{F}_{2},
\end{aligned}
$$

where $\psi_{1}$ and $\psi_{2}$ are stream functions for the upper and lower layer of fluids, respectively and $\mathscr{F}_{j}$ represents external forcing on the $j$ th layer, $J\left(f_{1}, f_{2}\right)=\nabla^{\perp} f_{1} \cdot \nabla f_{2}$

Received May 19, 2004; Accepted October 14, 2004

Address correspondence to Xiaoming Wang, Department of Mathematics, Florida State University and Iowa State University, Tallahassee, FL 32306, USA; E-mail: wxm@math.fsu.edu 
is the Jacobian,

$$
F_{j}=\frac{f_{0}^{2} L^{2}}{g\left(\frac{\Delta \rho}{\rho_{0}}\right) D_{j}}
$$

where

- $f=f_{0}+\beta y$ is the Coriolis parameter,

- $L$ is a typical horizontal length scale,

- $g$ is the gravitation constant,

- $D_{j}$ is the depth of the $j$ th layer,

- $\rho_{0}$ is the characteristic fluid density, and

- $\Delta \rho=\rho_{2}-\rho_{1}>0$ is the difference in density, and we consider a stably stratified case with lighter fluids (density $\rho_{1}$ ) sitting on top of heavier fluids (density $\rho_{2}$ ).

The boundary condition under consideration is the channel geometry, where all unknowns are periodic in the zonal (longitude $x$ ) direction with period $L$ and no penetration/no flow boundary condition in the meridional $(y)$ direction. More precisely, we have

$$
\begin{aligned}
\psi_{j}(x+L, y) & =\psi_{j}(x, y), \\
\psi_{j}(x, 0) & =\psi_{j}(x, h)=0 .
\end{aligned}
$$

Such a set of boundary conditions is more appealing than the usual doubly periodic boundary conditions. Physically the no-penetration in the latitudinal direction is closer to physics for flows on mid-latitude beta planes than the periodic boundary conditions, and the channel geometry (not the periodic geometry) allows us to derive conservation in time of the maximum modulus of the potential vorticity in the undamped/unforced environment(see for instance Chapter 1 of Majda and Wang, 2004, and Section 3 below).

Here we will consider the situation of a bottom layer much thicker than the top layer, i.e., $D_{2} \gg D_{1}$. Physically this could happen in many situations, for instance, in the ocean with a relatively thin (mixing) layer sitting on a much thicker (inertial) layer. The effective dynamics at large ratio of depth (bottom over top) is the socalled one and one-half layer model (See Eqs. 10, 11). This simplified effective dynamics was central to the successful quantitative prediction of the Great Read Spot on the Jupiter by Turkington et al. (2001). A natural question then is if such an approximation is valid. The purpose of this short note is to present a rigorous mathematical justification of this one and one-half layer quasi-geostrophic model from the two layer model (Eqs. 1, 2). More precisely, we demonstrate the following.

Theorem 1. For sufficiently smooth initial data $q_{j 0}, j=1,2$ and external forcing $\mathscr{F}_{j}$, $j=1,2$, the solutions $\psi_{j}, j=1,2$ of the two layer quasi-geostrophic equations $(1,2)$ converge to the solutions $\phi_{j}, j=1,2$ of the one and one-half layer quasi-geostrophic equations $(10,11)$ as $\varepsilon=\frac{F_{2}}{F_{1}}$ approaches zero. Moreover, the convergence rate is $\varepsilon$, i.e., for any fixed time $T$, there exists a constant $\kappa$ independent of $\varepsilon$ such that

$$
\left\|\psi_{j}-\phi_{j}\right\|_{L^{\infty}\left(0, T ; H^{2}\right)} \leq \kappa \varepsilon, \quad j=1,2 .
$$


The rest of this note is organized as follows. In Section 2 we formally derive the one and one-half layer model from the two layer model as the depth of the bottom layer goes to infinity. The rigorous mathematical justification of such a limit, i.e., the proof of Theorem 1, is presented in Section 3. We offer some concluding remarks and comments on the result obtained and some generalizations in Section 4.

\section{Formal Derivation}

Here we formally derive the one and one-half layer quasi-geostrophic model from the two layer quasi-geostrophic model assuming the bottom layer is much thicker than the top layer., i.e.,

$$
\frac{D_{1}}{D_{2}}=\varepsilon \ll 1 .
$$

Furthermore, we consider the case where the depth of the top layer and other parameters are fixed. Since the $F_{j}$ 's are inversely proportional to the depth of the layer with the other parameters fixed (see (3)), we may quantify this assumption of the disparity of the depth of the two layers in the relationship for the two layer model $(1,2)$ :

$$
F_{2}=\varepsilon F_{1}
$$

with $F_{1}$ fixed.

We may then rewrite (2) as

$$
\frac{\partial}{\partial t}\left(\Delta \psi_{2}+\varepsilon F_{1}\left(\psi_{1}-\psi_{2}\right)\right)+J\left(\psi_{2}, \Delta \psi_{2}+\varepsilon F_{1}\left(\psi_{1}-\psi_{2}\right)\right)+\beta \frac{\partial}{\partial x} \psi_{2}=0
$$

Formally setting $\varepsilon=0$ in the above equation and holding other parameters including $F_{1}$ fixed, we arrive at the following one and one-half layer quasi-geostrophic model as effective dynamics in this deep bottom layer case:

$$
\begin{gathered}
\frac{\partial}{\partial t}\left(\Delta \phi_{1}-F_{1} \phi_{1}+\beta y+F_{1} \phi_{2}\right)+J\left(\phi_{1}, \Delta \phi_{1}-F_{1} \phi_{1}+\beta y+F_{1} \phi_{2}\right)=\mathscr{F}_{1}, \\
\frac{\partial}{\partial t}\left(\Delta \phi_{2}+\beta y\right)+J\left(\phi_{2}, \Delta \phi_{2}+\beta y\right)=\mathscr{F}_{2} .
\end{gathered}
$$

We notice that the effective dynamics of the bottom layer is independent of the top layer and is governed by the barotropic quasi-geostrophic dynamics without topography.

On the other hand, we write

$$
h(\mathbf{x}, t)=F_{1} \phi_{2}(\mathbf{x}, t) .
$$

Then the effective dynamics of the top layer can be written as

$$
\begin{aligned}
\frac{\partial q}{\partial t}+J\left(\phi_{1}, q\right) & =\mathscr{F}_{1}, \\
q & =\Delta \phi_{1}-F_{1} \phi_{1}+\beta y+h .
\end{aligned}
$$


This means that a constant multiply of the bottom layer stream function serves as a (possibly time-dependent) effective topography for the top layer dynamics. This is precisely so in the case of the steady state (stationary) bottom layer stream function $\phi_{2}(\mathbf{x})$. Such steady states are easily achieved by appropriate choice of the forcing term $\mathscr{F}_{2}(\mathbf{x})$ dictated by this given stream function. Moreover, many of these kinds of stream functions are nonlinearly stable (see Majda and Wang, 2004, Chapter 4, among others).

The one and one-half layer model is intuitively correct. In the case of an extremely deep bottom layer, the inertia of the top layer is negligible when compared to that of the bottom layer, and hence the bottom layer should not feel the top layer.

An alternative scaling of the layers is to assume a fixed bottom layer depth and a vanishing top layer depth. In this case the parameters are

$$
F_{1}=\frac{F_{2}}{\varepsilon}
$$

with $F_{2}$ fixed.

The effective dynamics derived by formally setting $\varepsilon=0$ is then

$$
\begin{gathered}
\frac{\partial}{\partial t}\left(\psi_{1}-\psi_{2}\right)+J\left(\psi_{1}, \psi_{1}-\psi_{2}\right)=0 \\
\frac{\partial}{\partial t}\left(\Delta \psi_{2}-F_{2}\left(\psi_{2}-\psi_{1}\right)+\beta y\right)+J\left(\psi_{2}, \Delta \psi_{2}-F_{2}\left(\psi_{2}-\psi_{2}\right)+\beta y\right)=\mathscr{F}_{2} .
\end{gathered}
$$

We will not, however, pursue this limit here.

\section{Mathematical Justification}

Here we rigorously justify the one and one-half layer model as the limit of the two layer model $(1,2)$ as the ratio of the depth, i.e., $\varepsilon$, approaches zero.

\subsection{A Priori Estimates}

As in almost all rigorous work on perturbation problems, we need to derive uniform estimates in terms of the perturbation variable $\varepsilon\left(F_{2}\right)$. The natural way to derive such estimates is to utilize conserved quantities (in the unforced case) (see for instance Majda and Wang, 2004; Pedlosky, 1987; among others).

It turns out that the conserved quantity that is useful here is the maximum modulus of the potential vorticity in each layer in this channel geometry. We want to emphasize that the potential vorticities are conserved for our channel geometry but not necessarily conserved for the periodic geometry (see for instance Majda and Wang, 2004, Chapter 1, and the calculations below). Indeed, the two layer model $(1,2)$ can be viewed as the potential vorticities $q_{j}=\Delta \psi_{j}+(-1)^{j} F_{j}\left(\psi_{1}-\psi_{2}\right)+\beta y$ transported along each particle trajectory if there were no external forcing $\left(\mathscr{F}_{j}=0\right)$, and thus the maximum modulus of the potential vorticities remain the same along each particle trajectory. Moreover, for the channel geometry, trajectories that start within the channel never leave the channel, which ensures the conservation of the modulus of the potential vorticity. In our forced situation, we may multiply the equations by $q_{j}^{2 k-1}$, integrate over the channel, and apply Hölder's inequality to 
obtain

$$
\frac{d}{d t}\left(\frac{1}{2 k}\left\|q_{j}\right\|_{L^{2 k}}^{2 k}\right) \leq\left\|\mathscr{F}_{j}\right\|_{L^{2 k}}\left\|q_{j}\right\|_{L^{2 k}}^{2 k-1}
$$

where the nonlinear term drops out since

$$
\begin{aligned}
\int_{\Omega} \nabla^{\perp} \psi_{j} \cdot \nabla q_{j} q_{j}^{2 k-1} & =\frac{1}{2 k} \int_{\Omega} \nabla^{\perp} \psi_{j} \cdot \nabla q_{j}^{2 k} \\
& =\frac{1}{2 k} \int_{\Omega}\left(-\frac{\partial \psi_{j}}{\partial y} \frac{\partial q_{j}^{2 k}}{\partial x}+\frac{\partial \psi_{j}}{\partial x} \frac{\partial q_{j}^{2 k}}{\partial y}\right) \\
& =\frac{1}{2 k}\left\{-\int_{y=h} \psi_{j}(x, h) \frac{\partial q_{j}^{2 k}}{\partial x}(x, h)+\int_{y=0} \psi_{j}(x, 0) \frac{\partial q_{j}^{2 k}}{\partial x}(x, 0)\right. \\
& \left.\quad+\int_{x=L} \psi_{j}(L, y) \frac{\partial q_{j}^{2 k}}{\partial y}(L, y)-\int_{x=0} \psi_{j}(0, y) \frac{\partial q_{j}^{2 k}}{\partial y}(0, y)\right\} \\
= & 0
\end{aligned}
$$

where we performed integration by parts and utilized the channel boundary condition (5) in an essential way (the $q_{j}$ 's are not periodic in $y$ ), and the periodicity (with period $L$ ) of $\psi_{j}$ and $q_{j}$ in $x$.

Inequality (14) implies

$$
\left\|q_{j}\right\|_{L^{\infty}\left(0, T ; L^{2 k}\right)} \leq\left\|q_{j 0}\right\|_{L^{2 k}}+T|\Omega|^{1 / 2 k}\left\|\mathscr{F}_{j}\right\|_{L^{\infty}\left(0, T ; L^{\infty}\right)},
$$

which further implies

$$
\begin{aligned}
\left\|q_{j}\right\|_{L^{\infty}\left(0, T ; L^{\infty}\right)} & =\lim _{k \rightarrow \infty}\left\|q_{j}\right\|_{L^{\infty}\left(0, T ; L^{2 k}\right)} \\
& \leq\left\|q_{j 0}\right\|_{L^{\infty}}+T\left\|\mathscr{F}_{j}\right\|_{L^{\infty}\left(0, T ; L^{\infty}\right)} .
\end{aligned}
$$

Notice that this estimate is not uniform in $\varepsilon$ estimate for $\psi_{j}$. In order to get uniform estimates, we first derive a uniform estimate for the difference of the stream function by forming the difference of the two inequalities in (17). We have

$$
\begin{aligned}
& \left\|\Delta\left(\psi_{1}-\psi_{2}\right)-\left(F_{1}+F_{2}\right)\left(\psi_{1}-\psi_{2}\right)\right\|_{L^{\infty}\left(0, T ; L^{\infty}\right)} \\
& \quad=\left\|\Delta \psi_{1}-F_{1}\left(\psi_{1}-\psi_{2}\right)+\beta y-\left(\Delta \psi_{2}-F_{2}\left(\psi_{2}-\psi_{1}\right)+\beta y\right)\right\|_{L^{\infty}\left(0, T ; L^{\infty}\right)} \\
& \quad \leq\left\|q_{1}\right\|_{L^{\infty}\left(0, T ; L^{\infty}\right)}+\left\|q_{2}\right\|_{L^{\infty}\left(0, T ; L^{\infty}\right)} \\
& \quad \leq \kappa_{1}
\end{aligned}
$$

where we utilized simple triangular inequality and (17). Here and elsewhere, the $\kappa_{j}$ 's denote generic constants independent of $\varepsilon$.

The previous inequality implies, since $F_{1}+F_{2} \geq 0$,

$$
\left\|\Delta\left(\psi_{1}-\psi_{2}\right)\right\|_{L^{\infty}\left(0, T ; L^{\infty}\right)} \leq \kappa_{2}
$$


Combining this with the uniform estimates on the potential vorticity (17), we have

$$
\left\|\Delta \psi_{j}\right\|_{L^{\infty}\left(0, T ; L^{\infty}\right)} \leq \kappa_{3}, \quad j=1,2
$$

The global in time well-posedness of the two layer model follows from these two estimates together with the usual analysis for classical two-dimensional inviscid flows that can be found in Majda and Bertozzi (2001) among others. An alternative way to understand this is that, since the vorticities and potential vorticities remain bounded for all time, the Beale-Kato-Majda type criterion tells us that the classical solution must exist for all time.

We also claim that the same kind of estimates hold for the limit one and one-half layer model $(10,11)$. Moreover, since the bottom layer of the one and one-half layer model satisfies the barotropic quasi-geostrophic equation without topography, we can copy regularity works on two-dimensional Euler equations for incompressible perfect fluids and derive higher order estimates on the solutions (see Majda and Bertozzi, 2001, Section 4.4, and Temam, 1975, among others). This in turn implies higher order regularity on the top layer, since the stream function of the bottom layer serves as scaled effective topography for the top layer. In particular, we have

$$
\left\|\phi_{j}\right\|_{L^{\infty}\left(0, T ; H^{s}\right)} \leq \kappa_{4}, \quad j=1,2,
$$

for some sufficiently high index $s$.

\subsection{Convergence}

We are now ready to prove the convergence, i.e., Theorem 1.

We introduce the difference between the stream functions

$$
\tilde{\psi}_{j}=\psi_{j}-\phi_{j}, \quad j=1,2 .
$$

We first look into the equation satisfied by the difference of the bottom layer stream functions. This is expected to be relatively easier owing to the simplified dynamics for the bottom layer in the one and one-half layer model $(10,11)$. We then have, utilizing the two layer model $(1,2)$ and the one and one-half layer model $(10,11)$,

$$
\begin{aligned}
& \frac{\partial}{\partial t} \Delta \tilde{\psi}_{2}-F_{2} \frac{\partial}{\partial t}\left(\psi_{2}-\psi_{1}\right)+J\left(\psi_{2}, \Delta \tilde{\psi}_{2}\right)+J\left(\tilde{\psi}_{2}, \Delta \phi_{2}\right) \\
& \quad-F_{2} J\left(\psi_{2}, \psi_{2}-\psi_{1}\right)+\beta \frac{\partial \tilde{\psi}_{2}}{\partial x}=0
\end{aligned}
$$

Next we multiply this equation by $\Delta \tilde{\psi}_{2}$ and integrate over the channel. Notice that the nonlinear terms can be estimated, after performing elementary integration by parts, and utilizing (20) and (21) as well as Sobolev imbedding, as

$$
\begin{gathered}
\int \frac{\partial \tilde{\psi}_{2}}{\partial x} \Delta \tilde{\psi}_{2}=-\frac{1}{2} \int \frac{\partial}{\partial x}\left|\nabla \tilde{\psi}_{2}\right|^{2}=0 \\
\int J\left(\psi_{2}, \Delta \tilde{\psi}_{2}\right) \Delta \tilde{\psi}_{2}=\int \nabla^{\perp} \psi_{2} \cdot \nabla \Delta \tilde{\psi}_{2} \Delta \tilde{\psi}_{2}=\frac{1}{2} \int \nabla^{\perp} \psi_{2} \cdot \nabla\left(\Delta \tilde{\psi}_{2}\right)^{2}=0
\end{gathered}
$$




$$
\begin{aligned}
\left|\int J\left(\psi_{2}, \psi_{2}-\psi_{1}\right) \Delta \tilde{\psi}_{2}\right| & =\left|\int \nabla^{\perp} \psi_{2} \cdot \nabla\left(\psi_{2}-\psi_{1}\right) \Delta \tilde{\psi}_{2}\right| \\
& \leq\left|\Delta \tilde{\psi}_{2}\right|_{L^{2}}\left|\nabla \psi_{2}\right|_{L^{2}}\left|\nabla\left(\psi_{2}-\psi_{1}\right)\right|_{L^{\infty}} \\
& \leq \kappa_{5}\left|\Delta \tilde{\psi}_{2}\right|_{L^{2}}, \\
\left|\int J\left(\tilde{\psi}_{2}, \Delta \phi_{2}\right) \Delta \tilde{\psi}_{2}\right| & =\left|\int \nabla^{\perp} \tilde{\psi}_{2} \cdot \nabla \Delta \phi_{2} \Delta \tilde{\psi}_{2}\right| \\
& \leq\left|\nabla \tilde{\psi}_{2}\right|_{L^{4}}\left|\nabla \Delta \phi_{2}\right|_{L^{4}}\left|\Delta \tilde{\psi}_{2}\right|_{L^{2}} \\
& \leq \kappa_{6}\left|\Delta \tilde{\psi}_{2}\right|_{L^{2}}^{2} .
\end{aligned}
$$

Next, we need to estimate the time derivative of the difference of the stream functions of the top and bottom layer as suggested by (23). For this purpose, we take the difference of Equations (1) and (2) and we obtain

$$
\frac{\partial}{\partial t}\left(\left(\Delta-\left(F_{1}+F_{2}\right)\right)\left(\psi_{1}-\psi_{2}\right)\right)=-J\left(\psi_{1}, q_{1}\right)+J\left(\psi_{2}, q_{2}\right)+\mathscr{F}_{1}-\mathscr{F}_{2},
$$

which can be rewritten as

$$
\frac{\partial}{\partial t}\left(\psi_{1}-\psi_{2}\right)=\left(\Delta-\left(F_{1}+F_{2}\right)\right)^{-1}\left(-J\left(\psi_{1}, q_{1}\right)+J\left(\psi_{2}, q_{2}\right)+\mathscr{F}_{1}-\mathscr{F}_{2}\right)
$$

Thus, we have

$$
\begin{aligned}
\left|\frac{\partial}{\partial t}\left(\psi_{2}-\psi_{1}\right)\right|_{H^{1}} & =\left|\left(\Delta-\left(F_{1}+F_{2}\right)\right)^{-1}\left(-J\left(\psi_{1}, q_{1}\right)+J\left(\psi_{2}, q_{2}\right)+\mathscr{F}_{1}-\mathscr{F}_{2}\right)\right|_{H^{1}} \\
& \left.\leq \kappa_{7} \mid J\left(\psi_{1}, q_{1}\right)-J\left(\psi_{2}, q_{2}\right)-\mathscr{F}_{1}+\mathscr{F}_{2}\right)\left.\right|_{H^{-1}} \\
& \leq \kappa_{8}\left\{\left|\nabla \psi_{1}\right|_{L^{2}}\left|q_{1}\right|_{L^{\infty}}+\left|\nabla \psi_{2}\right|_{L^{2}}\left|q_{2}\right|_{L^{\infty}}+\left|\mathscr{F}_{1}-\mathscr{F}_{2}\right|_{H^{-1}}\right\} \\
& \leq \kappa_{9}
\end{aligned}
$$

where we have used (17) and the simple inequality

$$
\left|\int J(f, g) h\right|=\left|\int J(f, h) g\right| \leq|\nabla f|_{L^{2}}|g|_{L^{\infty}}|\nabla h|_{L^{2}}
$$

which implies that

$$
|J(f, g)|_{H^{-1}} \leq|\nabla f|_{L^{2}}|g|_{L^{\infty}} .
$$

Combining (24-27) and (30) and utilizing Cauchy-Schwarz we have

$$
\frac{1}{2} \frac{d}{d t}\left|\Delta \tilde{\psi}_{2}\right|_{L^{2}}^{2} \leq \kappa_{10}\left|\Delta \tilde{\psi}_{2}\right|_{L^{2}}^{2}+\kappa_{11} \varepsilon^{2}
$$

which further implies

$$
\left\|\psi_{2}-\phi_{2}\right\|_{L^{\infty}\left(0, T ; H^{2}\right)} \leq \kappa_{12} \varepsilon \text {. }
$$

This is the second estimate in Theorem 1. 
As a byproduct, we can estimate the time derivative of the difference of the bottom layer stream functions. This is needed in proving the convergence of the top layer, as we shall see below. For this purpose we go back to (23) and notice

$$
\frac{\partial}{\partial t} \tilde{\psi}_{2}=\Delta^{-1}\left(F_{2} \frac{\partial}{\partial t}\left(\psi_{2}-\psi_{1}\right)-J\left(\psi_{2}, \Delta \tilde{\psi}_{2}\right)-J\left(\tilde{\psi}_{2}, \Delta \phi_{2}\right)+F_{2} J\left(\psi_{2}, \psi_{2}-\psi_{1}\right)-\beta \frac{\partial \tilde{\psi}_{2}}{\partial x}\right)
$$

Thus we have, after applying elementary integration by parts and utilizing estimates (20), (21), (30), (32), and (34),

$$
\begin{aligned}
\left|\frac{\partial}{\partial t} \tilde{\psi}_{2}\right|_{H^{1}} \leq & F_{2}\left|\frac{\partial}{\partial t}\left(\psi_{2}-\psi_{1}\right)\right|_{H^{-1}}+\left|J\left(\psi_{2}, \Delta \tilde{\psi}_{2}\right)\right|_{H^{-1}} \\
& +\left|J\left(\tilde{\psi}_{2}, \Delta \phi_{2}\right)\right|_{H^{-1}}+F_{2}\left|J\left(\psi_{2}, \psi_{2}-\psi_{1}\right)\right|_{H^{-1}}+\beta\left|\frac{\partial \tilde{\psi}_{2}}{\partial x}\right|_{H^{-1}} \\
\leq & \kappa_{13} \varepsilon+\kappa_{14}\left|\nabla \psi_{2}\right|_{L^{\infty}}\left|\Delta \tilde{\psi}_{2}\right|_{L^{2}} \leq \kappa_{15} \varepsilon .
\end{aligned}
$$

We now proceed to prove the convergence of the top layer.

For this purpose we take the difference of the equations for the top layer in the two layer model (1) and that of the one and one-half layer model (10), and we deduce

$$
\begin{aligned}
\frac{\partial}{\partial t}\left(\Delta \tilde{\psi}_{1}-F_{1} \tilde{\psi}_{1}\right)= & -F_{1} \frac{\partial}{\partial t} \tilde{\psi}_{2}-J\left(\psi_{1},\left(\Delta-F_{1}\right) \tilde{\psi}_{1}\right)-J\left(\tilde{\psi}_{1},\left(\Delta-F_{1}\right) \phi_{1}\right) \\
& -J\left(\tilde{\psi}_{1}, F_{1} \phi_{2}\right)-J\left(\psi_{1}, F_{1} \tilde{\psi}_{2}\right)-\beta \frac{\partial \tilde{\psi}_{1}}{\partial x} .
\end{aligned}
$$

The estimates are very similar as for the bottom layer as given in (24-32). Indeed, we have, after multiplying (37) by $\left(\Delta-F_{1}\right) \tilde{\psi}_{1}$ and integrating over the channel,

$$
\frac{1}{2} \frac{d}{d t}\left|\left(\Delta-F_{1}\right) \tilde{\psi}_{1}\right|_{L^{2}}^{2} \leq \kappa_{16}\left|\left(\Delta-F_{1}\right) \tilde{\psi}_{1}\right|_{L^{2}}^{2}+\kappa_{17} \varepsilon^{2}
$$

where we have utilized the following inequalities which in turn rely on elementary integration by parts and estimates (20), (21), (34), (36),

$$
\begin{aligned}
\left|\int \frac{\partial}{\partial t} \tilde{\psi}_{2}\left(\Delta-F_{1}\right) \tilde{\psi}_{1}\right| & \leq \kappa_{15} \varepsilon\left|\left(\Delta-F_{1}\right) \tilde{\psi}_{1}\right|_{L^{2}}, \\
\int J\left(\psi_{1},\left(\Delta-F_{1}\right) \tilde{\psi}_{1}\right)\left(\Delta-F_{1}\right) \tilde{\psi}_{1} & =0 \\
\left|\int J\left(\tilde{\psi}_{1},\left(\Delta-F_{1}\right) \phi_{1}\right)\left(\Delta-F_{1}\right) \tilde{\psi}_{1}\right| & \leq \kappa_{18}\left|\left(\Delta-F_{1}\right) \tilde{\psi}_{1}\right|_{L^{2}}^{2}, \\
\left|\int J\left(\tilde{\psi}_{1}, F_{1} \phi_{2}\right)\left(\Delta-F_{1}\right) \tilde{\psi}_{1}\right| & \leq \kappa_{19}\left|\left(\Delta-F_{1}\right) \tilde{\psi}_{1}\right|_{L^{2}}^{2}, \\
\left|\int J\left(\psi_{1}, F_{1} \tilde{\psi}_{2}\right)\left(\Delta-F_{1}\right) \tilde{\psi}_{1}\right| & \leq \kappa_{20} \varepsilon\left|\left(\Delta-F_{1}\right) \tilde{\psi}_{1}\right|_{L^{2}}, \\
\int \frac{\partial \tilde{\psi}_{1}}{\partial x}\left(\Delta-F_{1}\right) \tilde{\psi}_{1} & =0 .
\end{aligned}
$$


The convergence of the top layer then follows from (38), namely,

$$
\left\|\psi_{1}-\phi_{1}\right\|_{L^{\infty}\left(0, T ; H^{2}\right)} \leq \kappa_{21} \varepsilon .
$$

This completes the proof of the theorem.

\section{Remarks and Comments}

We have established the validity of the one and one-half layer quasi-geostrophic model heuristically and rigorously from the two layer model. The convergence rate is $\varepsilon$, which is the ratio of the depth of the top thin layer over that of the thick bottom layer. The analysis is somewhat straightforward, since as we observe, the perturbation problem is a regular perturbation one (no initial, boundary, or internal layer/singularity emerges as the small parameter approaches zero). In fact, we can derive systematic asymptotic expansion of the solutions of the two layer model in terms of the small parameter $\varepsilon$. The systematic expansion demonstrates that the convergence rate of $\varepsilon$ is optimal. We will not pursue the details here.

Here we have discussed the situation of forced inviscid flows. Most physical problems involve both forcing and damping/dissipation. It seems that the analysis presented here may be generalized to certain special types of dissipation, for instance, for systems of the form

$$
\begin{aligned}
& \frac{\partial q_{1}}{\partial t}+J\left(\psi_{1}, q_{1}\right)=-d_{1}\left(\Delta \psi_{1}-F_{1}\left(\psi_{1}-\psi_{2}\right)\right)+\mathscr{F}_{1}, \\
& \frac{\partial q_{2}}{\partial t}+J\left(\psi_{2}, q_{2}\right)=-d_{2}\left(\Delta \psi_{2}-F_{2}\left(\psi_{2}-\psi_{1}\right)\right)+\mathscr{F}_{2},
\end{aligned}
$$

where the nonnegative coefficients $d_{j}$ 's are the analogs for the Ekman damping coefficient. Such nonsymmetric dissipations have been proposed by oceanographers (see, for instance, Holloway, 1992). The interested reader may work out the details. Nevertheless, it is still not clear how to handle the problem with classical (symmetric) Ekman damping, eddy viscosity, etc.

\section{Acknowledgments}

The authors acknowledge the financial support from NSF, DARPA, NAVY. Most of the work was done while the second author was visiting the Courant Institute of the Mathematical Sciences during the 2001-2002 academic year. Part of the work was done during the second author's visit to the Institute for Advanced Study at Princeton. The financial support and hospitality of IAS is graciously acknowledged.

\section{References}

Gill, A. (1982). Atmosphere-Ocean Dynamics. San Diego: Academic Press.

Holloway, G. (1992). Representing topographic stress for large scale ocean models. J. Phys. Oceanography 22:1033-1046.

Majda, A. (2002). Introduction to P.D.E.'s and Waves for the Atmosphere and Ocean. Courant Institute Lecture Notes Series. Amer. Math. Soc.

Majda, A., Bertozzi, A. (2001). Vorticity and Incompressible Flow. Cambridge: Cambridge University Press. 
Majda, A., Wang, X. (2004). Nonlinear Dynamics and Statistical Theories for Basic Geophysical Flows. Cambridge, UK: Cambridge University Press.

Pedlosky, J. (1987). Geophysical Fluid Dynamics. 2nd ed. New York: Springer-Verlag.

Temam, R. (1975). On the Euler equations of incompressible perfect fluids. J. Funct. Anal. 20(1):32-43.

Temam, R. (2000). Navier-Stokes Equations. Rhode Island: AMS.

Turkington, B., Majda, A. J., Haven, K., DiBattista, M. (2001). Statistical equilibrium predictions of jets and spots on jupiter. Proc. Natl. Acad. Sci. USA 98(22):12346-12350. 